\title{
Laboratory Environment and Senior Secondary Two Chemistry Students' Performance in Quantitative Analysis
}

\author{
${ }^{1}$ Dr. Nsikak-Abasi Udofia $\&^{2}$ Nsikanabasi Udo Obot \\ ${ }^{1}$ Department of Educational Foundations, University of Uyo, Uyo, Akwa Ibom State \\ ${ }^{2}$ Institute of Education, University of Uyo, Uyo, Akwa Ibom State
}

\begin{abstract}
This study investigated laboratory environment and senior secondary two chemistry students' performance in quantitative analysis. Specifically, it examined how group and individual session participation; efficient laboratory assistant and non-efficient laboratory assistant; laboratory apparatus and non-quantitative apparatus; and teacher's attitude affects academic performance in quantitative analysis. The population of the study consisted of six hundred and fifty (650) senior secondary students' in Public schools in Ikot Ekpene Local Government Area from which a stratified random sampling technique was used to select thirty (30) students in the study area. Quasi-experimental design was used in this study. The instrument for data collection was Practical Chemistry Achievement test (PCAT). The PCAT gave a reliability coefficient of 0.99. The results obtained gave a significant difference between laboratory apparatus and non-laboratory apparatus; and teacher's attitudes with academic performance in quantitative analysis. Equally no efficient laboratory assist; group and individual practical session participation with academic performance in quantitative analysis. On the basis of the findings, it was recommended that students, teachers and government should improve the effective use of laboratory environment to foster Senior Secondary Schools' Chemistry students' performance in quantitative analysis.
\end{abstract}

Keywords: Laboratory environment, Chemistry students, Performance in Chemistry, Quantitative analysis

\section{Introduction}

Science is the pursuit of knowledge arranged in an orderly manner, obtained by observation and testing of facts. Following from this, Chemistry may well be regarded as one of the main braches of pure science. It deals with the ways in which man obtains the material needed for survival. It also deals with the materials comprising the universe; for instance land, water, air, sky etc, and the ways these materials behave when in contact with each other.To determine the composition of substances in chemistry, analysis is usually done qualitatively or quantitatively.

1. Qualitative analysis:Used to identify the elements and compounds that are present in a sample of a given element.

2. Quantitative analysis: Used to calculate the amount or quantity of an element or compound present in a given sample. The ultimate aim of a quantitative analysis is to ascertain 'how much' of a given species is present in a sample of matter; depending upon the procedure employed, this may be accomplished directly or very indirectly.

Quantitative analyses are conveniently classified into the following methods according to the nature of its final measurement:

(a) Gravimetric analysis: If it consists of securing the weight of a solid.

(b) Volumetric analysis: If it involves determination of a volume.

(c) Colorimetric analysis: If the absorption of light is measured

(d) Electro analytical: If electrical property is determined.

Steps common to all quantitative analytical methods

Planning the analysis:The analysis may involve what you do not know hence you need to get references:

1. What information is required by the time the work is done?

2. What analysis will be done to give the required information?

3. What methods will be used (instruments)?

4. How many samples will be analysed; because the number of samples will determine the method to be able to perform the analysis on time.

Sampling:This is how to obtain the materials because the samples must be a representation of the actual materials. This can be done through: 
1. Gross sampling

2. Random sampling

Drying the sample:Drying is carried out to free the sample from moisture. This is done within $100-110^{\circ} \mathrm{C}$. Drying will remove absorbed water but not chemically absorbed matter (water of crystallization). For substances that decompose, a temperature of $60^{\circ} \mathrm{C}$ is used.

Weighing the sample:A sensitive balance is used; a pipette or burette is used to take little amounts of substance. Weighing can be done through:

1. Direct Weighing

2. Weighing by deference

Dissolving the sample:Usea suitable solvent if the sample is known.Ifthe sample is unknown, trial and error methods areemployed but whatever solvent used, it should:

1. Completely dissolve the sample

2. It should not pose a problem (interfere) in any subsequent step of the procedure.

Separation of interferers:This is achieved through:

1. Precipitation

2. Masking

3. Electrode deposition

Measurement of the sample: All of the preliminary steps in an analysis are undertaken in order to make a final measurement at a true gauge of the quantity of the species being determined.

Calculation and interpretation of results: Since the information desired from the analysis is usually extensive in nature, it is necessary to relate mathematically the values of the final measurement to the weight of the sample employed in its procurement.

Finally, the report of quantitative analysis is incomplete without some indication being given regarding the reliability of the results (Singh, 1976).

Chemistry as an experimental science needs a place where the findings about the universe are carried out, the laboratory. A chemistry laboratory can be said to be a place fitted with adequate materials for experimentations and demonstrations. These materials include the laboratory fixtures: benches, cupboards, apparatus and chemicals. The laboratory experiment could be said to be an instructional procedures in which cause and effect, nature or property of any object or phenomenon is determined by individual experience generally under controlled conditions.

It is obvious that the study of chemistry is influence by curiosity and constructiveness, which can be satisfied by the opportunity provided by it. The desire to do a particular thing in order that something may happen appears to be the starting point for experimental work for those offering the subject. Certainly, more benefits obtained by the students from effective teaching of chemistry are 'care' and 'skill' in handling apparatus and chemicals and the use of correct method in obtaining accurate experimental results.

Therefore, a laboratory mainly set up for teaching of chemistry in any level of study must be well equipped with materials for experimentation, in order to arouse the curiosity and willingness to study. It is on this basis that this research is undertaken with emphasis on laboratory influence on students' attitude and academic performance in quantitative analysis.

It is worthy to note that at post-primary school stage, chemistry is aimed at:

1. Exposing the students to the basis chemical concepts.

2. Individuals experimentation using simple in-expensive equipment

3. The role and significance of chemistry in the study of other disciplines for instance-biology, agriculture science, medicine and pharmacy.

4. The relevance of chemistry to the society

5. Extracurricular activities designed to inculcate a deeper appreciation of science and technology and their role in the development of human societies etc.

Although practical work in chemistry may be termed 'an organized common sense', it has a technique which is too often neglected. Laboratory technique can gradually be built up by the best use of both demonstration and individual experiments. The students may encounter the difficulties of developing the laboratory techniques and the command of language. Here the demonstration serves as an essential purpose for this course as it can be used as an exhibition lesson to point out the essentials of the experiment. Also, it shows the correct use and limitations of chemicals, hence initiated into broad principles of laboratory technique. 
At individual level, students learn by doing, but they must know the type of apparatus and the chemicals to use before they can work with the minimum instructions. Infact, the students alone cannot be expected to know the distinctive uses of reagent; for instance tetraoxosulphate(vi) acid, sodium hydroxide etc. even the correct amount of chemicals to be used in a particular experiment usually require practice and instructions. Difficulties of this kind must always be kept in mind and the relative advantages of each kind of chemical must be demonstrated. But where these important materials are not available in the laboratory, the performance of students in quantitative analysis becomes a problem because teaching cannot be effective.

At this point in time, it is worth mentioning here that schools should provide the facilities needed and equip their laboratory so that students can be curious to know the usage of this equipment thereby improving their attitude and performances in quantitative analysis. Again, schools offering chemistry should have a laboratory assistant whose duties to care for the chemicals/apparatus in the laboratory. Here a strong relationship between teacher/students/assistants is emphasized. Since the essential starting points in chemistry are vested primarily in clear and good experimental technique followed by unambiguous chemical language, the researcher, therefore, gathered the challenge to aggregate facts about laboratory essential role in influencing student's attitude and academic performance in quantitative analysis.

Science is concerned with interpretation of nature and natural events; knowledge employed for such interpretation is usually acquired systematically.The major difference between an ordinary classroom and a chemistry laboratory is the presence of apparatus/chemicals in the latter for student's activities. A laboratory without the chemicals or apparatus cannot therefore meet the purpose of which it is built.

According to Ausubel (1968), the objectives of the practical works therefore are the objectives of the laboratory. The basic functions of the laboratory are in computerizing experiences using objects. The laboratory gives the students an appreciation of the study of science and thus promoting problem in solving, analytical and generalizations abilities and thus providing the students with the understanding of science.

Schulman (1973), Robinson, (1969); benzuictal, (1977), in their study of the laboratory science, stated that laboratories are used in science to arouse and maintain interest, attitudes, satisfaction, open-mindedness and curiosity. He further maintains that laboratories in science are used to develop creative thinking and problem solving, to develop practical abilities and the promotion of scientific thinking and hypothesis formulations and assumptions.

Another major researcher in the laboratory science Anderson (1978) claims that what goes on in the laboratory is designed to foster knowledge of human enterprise in science so as to enhance students intellectual and effective understanding; to foster science inquiry skills that can be transferred from one sphere of problems solving to another. He further ascertains that the laboratory work helps the students appreciate impact and evaluate the role of science and to help students grow both in appreciation of the orderliness of scientific knowledgeand also in understanding the tentative nature of scientific theories and models.

Onwioduokit (1989), a strong proponent of laboratory science concluded that the laboratory work is meant to develop critical and analytical thinking in students under a problem solving situation and to inculcate problem solving skills such as manipulative, cognitive, cognitive, observational, computational and communicative. Abdullahi (1982) states that the laboratory work is desirable because it yields some desirable skills such as the ability to plan an experiment and analyse the practical problem into its component parts, the ability to perform experiment and the ability to interpret the results of experiment and draw inferences and conclusions. Chemical solutions according to UNESCO (1973:29), are materials dissolved in water (or other solvents) that is of interest. It is the dissolved material that is chemically changed. Davis, Dennial, Locke and Reay (1973) recognized acids, alkalis and salts as the three important chemicals frequently used in the laboratory work. The common laboratory acid according to them is tetraoxosulphate(vi)acid, Ethanoic acid, hydrochloric acid, trioxonitate(v)acid etc. Noibi (1984), stated that laboratory work helps students to achieve and develop the seven forms of competences which the classed as vocabulary, learning skills, methods of inquiry, perspective, limitations and sensitivity.

From the above analyses, coupled with the principles of science teaching, the researcher concludes that it is most probable that students learn more from science lessons when they are given the opportunity to learn through doing than when they are simply allowed to observe. With current decline in finance, many schools cannot have all the chemicals/equipment needed to run the laboratory. Some schools have chemicals which have not been put too much use and some are deteriorating. This means a waste of scare commodities.

Equally, student's poor academic performance is science subjects at the senior certificate and the National Examination Council (NECO) examination has continued to pose serious concern to educators, parents and the government, when considered against the scientific and technological take-off.

Previous research studies have attributed this problem of poor academic performance of students in quantitative analysis to students study habits, self-concepts, attitude, poorly equipped laboratories, lack of efficient laboratory assistant and the teacher-related variables of qualification and years of teaching 
experience.This study therefore sets out to find out laboratory influence on students attitude and academic performance in quantitative analysis in Ikot Ekpene Local Government Area.

\section{Research Questions}

i. Is there any significant difference between group and individual practical session participation on academic performance of students in quantitative analysis?

ii. Is there any significant difference between efficient laboratory assistance and non-efficient laboratory assistant on academic performance in quantitative analysis?

iii. Is there any significant difference between teacher's attitude and academic performance in quantitative analysis?

\section{Research Hypotheses}

The following null hypotheses were tested at 0.5 level of significance:

i. There is no difference between group and individual practical session participation with academic performance in quantitative analysis.

ii. There is no significant difference between efficient laboratory assistant and non-efficient laboratory assistant with academic performance in quantitative analysis.

iii. There is no significant difference between teacher's attitude and academic performance in quantitative analysis.

\section{Methodology}

Design: The quasi-experimental design was adopted in this study. This design allowed for the testing of research hypotheses based on cause and effect relationship between independent and dependent variables. In this design, the researchers deliberately controlled and manipulated the conditions which determinedthe events the research was focusing on.

Population: The population for this study comprised of Six Hundred and Fifty (650) Senior Secondary Two (SS2) science students in public schools Ikot Ekpene Local Government Area of Akwa Ibom State in the 2006/2007 academic session.

Sampling: A stratified random sampling technique was used to select students in the research area. The samplefor the study was consisting of Thirty SeniorSecondary Two (SS2) Students in Ikot Ekpene Local Government Area in the 2006/2007 academic session. Thisclass was selected because it is presumed that students at this level would have a fair exposure to chemistry concepts.

Instrumentation:A standardized chemistry test was issued out to these selected students. The appropriate test items were written and edited. A copy of all the items in the test and format was presented to the research's supervisor and a colleague in the teaching fields. Certain items in the test which were not found suitable or were considered as repetitions were dropped. All comments and suggestions by the supervisor and the colleague were incorporated in the validities of the instrument. The reliability of the instrument was 0.99. The test-retest method was used to estimate the reliability of test scores. This method involves administering the same test to Thirty (30) students who were not part of the study on two separate instances. The reliability coefficient was 0.99 .

Procedure for data collection: The researchers obtained permission from the authorities of the school used in the Local Government Area. A micro teaching on volumetric analysis was carried out before the administration of the students. The test was administered directly to the students sample after the students were divided into two sets: individual working students and students working in groups. For instance, hypothesis two the independent variables in the experiment are efficient laboratory assistant and non-efficient laboratory assistant which the researchers manipulated to determine their effect on students' academic performance (dependent variable) in quantitative analysis. The experimental or treatment group which comprised individual students who were exposed to an efficient laboratory assistant and the control group which were students in groups (five students made up a group) serves as a baseline against which to compare the group receiving the experimental treatment. The extraneous variable was built into the study as an independent variable. The method helped the researcher extract from the total variance of the dependent variable, the variance due to the variable. The same procedure was incorporate to obtain values for the subsequent hypothesis in this research study.The test was collected immediately after responses by the students.There was no need to explain the items to them because the students at this level would have fair exposure to basic chemistry concepts. Therewas only an appeal for object and sincere response was made to them. 
Thedata collected were scored and analyzedby the researcher. The test questions set to evaluate the students covered the cognitive domain of educational objectives. The test also took into cognizance the research questions and hypothesis. The t-test (paired comparison test) was the technique used to test the hypothesis.

\section{Results}

Table 1: Group and individual practical session participation with academic performance in quantitative

\begin{tabular}{lllllll}
\multicolumn{7}{c}{ analysis } \\
\hline Students & Score A & Score B & $\boldsymbol{\Sigma} \mathbf{d}$ & $\mathbf{\Sigma d}^{\mathbf{2}}$ & $\mathbf{t}_{\text {cal }}$ & $\mathbf{t}_{\text {tab }}$ \\
\hline 1 & 28 & 28 & 0 & 0 & & \\
2 & 25 & 27 & -2 & 4 & & \\
3 & 27 & 27 & 0 & 0 & 1.19 & 2.78 \\
4 & 27 & 23 & +4 & 16 & & \\
5 & 27 & 21 & 6 & 36 & & \\
& & & 8 & 56 & & \\
\hline
\end{tabular}

Table 1 shows that the calculated test statistics value (1.19) is less than the tabulated test statistics value (2.78) at degree of freedom $(\mathrm{df})=1$ and 0.05 level of significance. The null hypothesis was accepted. The result shows that there is no significant difference between group and individual practical session participation with academic performance in quantitative analysis.

Table 2: Efficient laboratory assistant and non-efficient laboratory assistant with academic performance in

\begin{tabular}{lllllll}
\multicolumn{7}{c}{ quantitative analysis } \\
\hline Students & Score A & Score B & $\boldsymbol{\Sigma} \mathbf{d}$ & $\mathbf{\Sigma} \mathbf{d}^{\mathbf{2}}$ & $\mathbf{t}_{\text {cal }}$ & $\mathbf{t}_{\text {tab }}$ \\
\hline 1 & 28 & 27 & 1 & 1 & & \\
2 & 24 & 29 & -5 & 25 & & \\
3 & 23 & 26 & -3 & 9 & -0.93 & 2.78 \\
4 & 22 & 26 & -4 & 16 & & \\
5 & 28 & 24 & +4 & 16 & & \\
& & & -8 & 67 & &
\end{tabular}

Table 2 shows that the calculated test statistics value $(-0.93)$ is less than the tabulated test statistics value (2.78) at degree of freedom (df) $=1$ and 0.05 level of significance. The null hypothesis was not rejected. The result is that there is no significant difference between efficient laboratory assistant and non-efficient laboratory assistant with academic performance in quantitative analysis.

Table 3:Teachers' attitude and academic performance in quantitative analysis

\begin{tabular}{lllllll}
\hline Students & Score A & Score B & $\mathbf{\Sigma d}$ & $\mathbf{\Sigma d}^{\mathbf{2}}$ & $\mathbf{t}_{\text {cal }}$ & $\mathbf{t}_{\text {tab }}$ \\
\hline 1 & 20 & 20 & 0 & 0 & & \\
2 & 28 & 29 & -1 & 1 & & \\
3 & 20 & 20 & 0 & 0 & & \\
4 & 27 & 26 & 1 & 1 & 3.64 & 2.26 \\
5 & 29 & 25 & 4 & 16 & \\
6 & 25 & 24 & 1 & 1 & \\
7 & 27 & 26 & 1 & 1 & \\
8 & 27 & 26 & 1 & 1 & \\
9 & 28 & 27 & 1 & 1 & \\
10 & 29 & 27 & 2 & 4 & \\
& & & 10 & 26 & \\
\hline
\end{tabular}

Table 3 shows that the calculated test statistics value (3.64) is greater than the tabulated test statistics value (2.26) at degree of freedom ( $\mathrm{df})=1$ and 0.05 level of significance. The null hypothesis is rejected. The result is that there is a significant difference between teachers' attitude and academic performance in quantitative analysis.

\section{Discussion of Findings}

The discussion of results based on the research questions and hypothesis formulated for the study and conclusion arrived at after careful analysis of the data from Senior Secondary two students (SS2) from the secondary school in the area under the study.Therefore, a laboratory without reagents cannot therefore meet the purpose for which it is built.Table 1 shows a paired comparison test value that $\mathrm{t}_{\text {cal }}$ is 1.19 (critical value at 0.5 level of significance of 2.13) the result is thatthere is no significant different between group and individual practical session participation with academic performance in quantitative analysis.

The chemistry curriculum recommends the guided discovery approach, resting on the activityof the pupils be used in teaching. Wopfer, (1977) stated that a student who has acquired basic manipulative skills should be able to perform common laboratory techniques with care and safety.Ausubel (1963) opined that 
meaningful learning occurs when there is interaction between the student's appropriate elements, in the science knowledge that already exists and the new materials to be learned. Where such interaction does not exist, rote learning is said to have occurred. Brunner (1960), in his theory supports the promotion of acquisition of knowledge through discovery learning. On the other hand; Gagne, (1965) is of the opinion that students based on subsequent prerequisite knowledge can acquire knowledge.

Prior knowledge in science subjects determines what further learning should take place. Individual participation of students in the practical session provides opportunity for creative children to act out since they are not restricted at all which could have hindered them (students) from showing their creativity; it enables the measurement of higher cognitive levels of the individual students in practical session gives room for cheating, difficult to measure higher cognitive levels of those students and correct answers to practical group work cannot be inferred that those students know the answer. Recent trends in science teaching call for units of exercise involving individual intimate participation of the student experimentation with familiar materials, observation and analysis. Where the students are mainly guided to explore for him, the learning process is more interesting and the students is given opportunity to develop some skill in manipulation of equipment (STAN, 1978). The value of laboratory works as a means of involving students concrete experience with object value and concept has been suggested and emphasized [Ausubel, 1968]; $\quad$ Babikan, (1970); Abdullahi (1982). Onwioduokit (1989) re-echoes that students many know best about those things with they had direct experience and least about those, which they have memorized.

If students are passive listeners, individual participation in the teaching learning session, they will not be involved in the active and participatory learning and will also be no development of student's self-esteem and self-confidence free from the biases of teachers and parents and also no development to the fullest, their innate potentials (Etuk, 2006)

Table 2 shows a paired comparison test value of $t_{\text {cal }} 0.925$. The result gives no significant different between efficient laboratory assistant and non -efficient laboratory assistance with academic performance in quantitative analysis.The result contradicts Eshiet (1993) who stated that it is necessary to have trained laboratory assistants and technicians in every laboratory. This contrast may be due to insufficient apparatus which resulted in grouping of students and the laboratory assistants inability to contend with the different students in group during the practical session; equally, the laboratory assistants had to be dispelling the reagents at different intervals when the quantity was insufficient to the students and the laboratory assistants admonishing the students in groups work who exhibited laissez-faire attitude during the practical session to get involved or contributed to the completion of the practical session none-efficient laboratory assistant are not helpful in setting up apparatus for demonstration or normal class work. Most teachers who had little or no knowledge of the designs of new equipment spent much time mastering how to use them and carry out repairs on minor faults and equally, teachers need time to seek for new ideas through research hence, there is need for an efficient laboratory assistant - Eshiet, (1993).

Table 3 shows a paired comparison test value $\mathrm{t}_{\text {cal }} 3.64$. This results indicates that there is a significant different between teachers attitude and academic performance in quantitative analysis. This means that teachers with positive attitude in teaching chemistry will invariably influence the student's attitude positively. This result is expected and not surprising because teacher's attitude as perceived by students affects to a large extent the student's attitude toward the teacher and academic achievement in quantitative analysis. If a teacher presents his/herself in a friendly manner in the class, interest is aroused in the learner with the consequent attention to what he present in the class, but for the contrary, the learner would view the teachers attitude as non-informative leading to inhibition of attention and learner's negative emotion inhibition their academic achievement.

These findings are supported by the work of Ecewu (1981) who posited that the behaviour of pupils is a function of teacher's personality and classroom behaviour. However, the findings by Fuchs (1968) contradict the present work. According to him, educational problems lie with parents and that blame on teachers is not necessary. This assertion may be due to careless attitude of some parent towards their children's education especially in the areas of provision of textbooks to the students.

Equally, the teacher's ability to choose, select and use teaching resources (i.e. those things which aid or promote the student's learning) under varying situation so as to make his/her teaching productive and to communicate that are incidental to the process of teaching $d$. since facts and information are more readily retained when supplemented with teaching aids, the chance are that if materials are carefully selected and skillfully used, learning will be move meaningfully (Adeniyi, 1981). In essence, without the teacher, the will be no implementation of the curriculum.

\section{Conclusion}

The result of the analysis of the collected data show that is a strong influence of laboratory environment on students' performance in quantitative analysis and this is very important in enhancing of manipulative skills.A significant difference was obtained between laboratory apparatus and non-laboratory 
apparatus with academic performance in quantitative analysis and also between teacher's attitude and academic performance in quantitative analysis. These attest to laboratory apparatus and reagent being the import contents deciding whether a laboratory is standard or not and equally, the relevance of a teacher as the implementer of the curriculum for the success of the learning process.

No significant differences were obtained between group and individual practical session participation with academic performance in quantitative analysis and also between efficient laboratories assistant and nonefficient individual practical session participation is recommended to enhance proficiency in manipulative skills and allows creative student's to act out instead of being in group work which inhibits innate potentials.

However, the data obtained for efficient laboratory assistance and non-efficient laboratory assistant contradicts the relevance of an efficient laboratory assistant but infact the duties and role of a laboratory assistant as an important personnel in a laboratory is highly emphasized even in this research study for the sustainability of a standard laboratory and the teaching process in quantitative analysis.

\section{Recommendations}

Based on the findings of the study, the following recommendations are made:

1. Availability of laboratory apparatus and reagents are essentials in a functional laboratory. The science teacher who is efficient, effective and creative, for day-to-day running of the laboratory, should keep accurate records of a stock book which should contain all the apparatus and reagents. This will enable replenishing of exhausted supplies of reagents

2. Individual participation of students in practical sessions should be adopted to create opportunities for student-material interaction, which will enhance development of higher cognitive abilities such as application, analysis, synthesis and evaluation.

3. Education boards should organize workshop/seminars for both chemistry teachers and laboratory assistance during vacations to keep them in touch with modern teaching methods.

4. Well-qualified science teachers and laboratory assistants should be employed at the federal, state and local government levels and there cognizance in the selected of teaching strategies and learning materials.

5. Chemistry teachers should adopt strategies that will enhance the development of positive attitude to quantitative analysis among students.

\section{References}

[1]. Abdullahi, A. (1982) Science Teaching in Nigeria. Atoto Press Limited, Ilorin.

[2]. Adeniyi, A. B. (1981): Improvisation in the Chemistry laboratory. Journal of Science Teachers' Association of Nigeria (STAN). Vol. 19, No 3, P. 57-62.

[3]. Allison, M. T. (1977): A Modern approach to W.A.S.C. Practical Chemistry Examination. Journal of Science Teachers' Association of Nigeria (STAN) Vol. 15, No. 3 P. 121-125.

[4]. Asubel, D. P. (1968): The Use of Advance Organizer in the Learning and Retention of Meaningful Verbal Material. Journal of Education Psychology. 51:p.267-272.

[5]. Babikan, Y. G. (1970) and Burmastor, M. A. (1953): In Akusoba, E. U. "The Secondary School Chemistry teacher perception on the goals of laboratory activities and the skills students should derive from them"Journal of Science Teachers Association of Nigeria (STAN). Vol. 23, No. 1 and 2 (Dec., 1985) P. 131-140.

[6]. Bruner, J. S. (1960): The process of Education. New York. Alfred A. Knof Inc.

[7]. Davis, L., Dennial, Lucke and Reay (1973) Investigating Chemistry.

[8]. Eshiet, I. T. (1993) Science Laboratory Organization and Management. Nigeria: Belpot Inc.

[9]. Eshiet, I. (Ed). Methodology of Science teaching (Historical and Conceptual Approach). Nigeria: Belpot Inc.Co.

[10]. Etuk, N. E. (2006): Students-Centred Teaching: A Holistic Approach to Teaching for Effective Learning. [A discussion Guide]

[11]. Ezewu, E. E. (1981): Social Psychological Factors of Human Learning in School. Onitsha; Leadway Books Publishers.

[12]. Gagne, R. M. (1965): The Condition of Learning. New York.

[13]. Ihde, J. A. (1963): “The Development of Science Instruments in the Seventh Century". A Paper Presented at a History of Science Symposium. New York, Double Anchor Books.

[14]. Njoku, Z. O. (1990): Competencies need and Possessed by Science laboratory Assistants in Anambra State Secondary Schools. Unpublished M. Ed. Thesis, Dept. of Education, University of Nigeria, Nsukka.

[15]. Noibi, A. S. (1984): "Personal resource for Science Teaching". A paper Presented at Science Teachers Association of Nigeria Conference at Unical.

[16]. Onwioduokit, F. A. (1989): "Development of Student's Laboratory Skills Using three problem-solving Model in Practical Physics". Ph.D. Thesis (unpublished.). Universityof Ibadan, Nigeria.

[17]. Otuka, J. O. E. (1981): “The Ordering Stocking and Storing of Laboratory Equipment". Journal of STAN. Vol. 19 No. 3.

[18]. Robinson, T. J. (1969) and Benzuietal (1977): In Akusoba, E. U.The Secondary School Chemistry Teachers Perception on the Goals of laboratory activities and skills student should derive from them" (STAN) Vol. 23, No. 1 and 2 (Dec., 1985) P. 29-36.

[19]. Singh, K. (1975): Chemical Analysis for M.Sc. Analytical Chemistry Students. Ahmadu Bello University Zaria, Nigeria. 